human feeder cells or a no-feeder cell protocol and culture media without animal serum or proteins. Although not currently available, Nakatsuji speculates that such clinical-grade human embryonic stem cells will be obtainable within a few years. But the future existence of these cells is still not the panacea some may think it is.

"Just injecting stem cells is not going to work," said Shahin Rafii, a physician and stem cell scientist at Cornell University Medical College. "First, you have to be able to differentiate the cells into functional, transplantable tissues. We don't really know how to do this yet."

Directing the differentiation of the cells is just one obstacle. Scientists also do not know whether differentiated patientspecific stem cell lines would be immunologically tolerated by the donor/host or whether they would be safe or effective. Yet another problem is that stem cell lines created from patients with diseases will carry features of the disease and would likely not be useful for treating those patients.

Beyond difficulties with the cells themselves, the adult tissue environment where the cells need to thrive also hinders the progression to clinical transplantation. "For cell therapy to work you need to be able to get the cells to engraft and expand after transplantation," Marcus Grompe, a hepatic stem cell researcher at Oregon Health \& Science University, told the JCI. "That part is very difficult and has not been solved at all for some key tissues ... we are still several years away from clinical trials."

In order to be useful for treating neurodegenerative diseases, for example, the stem cells need to survive in the damaged and diseased brain, make proper synaptic connections, and form appropriate pathways for proper neural function. "We have no idea - at least not yet - of how to accomplish this feat," said Steve Goldman, chief of the Division of Cell and Gene Therapy at University of Rochester Medical Center.

"Making matters worse," Goldman explained, "once a desired cell type is generated from an embryonic stem cell, its isolation to appropriate levels of purity is by no means

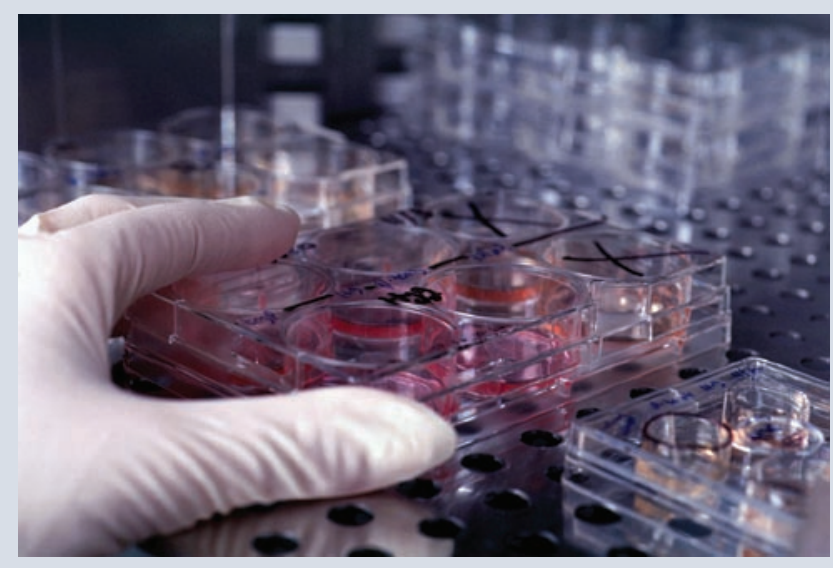

Culture trays containing human embryonic stem cells. Photo courtesy of James Thomson and Jeff Miller (University of WisconsinMadison, Madison, Wisconsin, USA).

straightforward." Isolating pure stem cells is not foolproof, and remnant cells typically persist. These cells can generate a whole host of tumors even if they are completely diploid and genomically stable.

Another issue, according to Rafii, is proper vascularization of these transplanted cells to ensure that their blood supply is adequate. Rafii said that it may be necessary to nanofabricate small blood vessels and put these into the cells before transplantation.

Although no miracle cures have been found yet, these obstacles are not cause for pessimism. According to Charles Jennings, executive director of the Harvard Stem Cell Institute, "It is a fast-moving field. Human embryonic stem cells were just isolated in 1998. It's still early."

\section{Stacie Bloom}

\title{
Prominent investigator wins diabetes research award
}

hilipp Scherer was presented with the American Diabetes Association's 2005 Outstanding Scientific Achievement Award on June 13, 2005. Scherer is an associate professor in the Department of Cell Biology \& Medicine at Albert Einstein College of Medicine. He spoke with the JCI about this award, his research, and his career.

JCI: Which of your accomplishments do you feel led to this award?

Scherer: We are probably best known for our work on the adipocyte-specific secretory protein adiponectin/Acrp30.
This protein has proven to be an excellent biomarker for systemic insulin sensitivity, and also a predictive marker for cardiovascular risk in a large variety of patient populations. Beyond its use as a marker in the clinical setting, this protein is directly implicated as a potent insulin sensitizer and antiatherosclerotic mediator.

I like to think that my group has had an impact in other areas of adipocyte biology as well. We have further established the role of adipose tissue as an endocrine organ through the description of physiological effects of an array of adipocyte- derived secretory products (adipokines) that include resistin, acute phase reactants, and proinflammatory cytokines as well as the involvement of the p38 MAPK pathway during adipogenesis.

JCI: What do you think is the most important discovery you have made?

Scherer: The initial cloning and analysis of adiponectin. We isolated Acrp30 in 1993 while I was a postdoc in Harvey Lodish's laboratory at the Whitehead Institute, and we published these findings in 1995. Additional groups described this protein subsequently in 1996, and it was known by 
a variety of names until our Japanese colleagues suggested the name adiponectin in 1999. Adiponectin received very little attention for several years because it is challenging to express in a recombinant form and it was difficult to disrupt the adiponectin locus. It wasn't until 5 to 6 years after the initial description that the field gained momentum, and by now more than 1,000 publications deal with the topic.

JCI: What, if anything, do you consider to be the turning point of your career?

Scherer: I don't believe that a single event or a single "spectacular gel" can turn an entire career around. However, I do believe that over a significant amount of time, enough momentum can be achieved to make a significant contribution to the field. For my laboratory, several years of work from a number of lab members have resulted in a publication in 2001 in which we described some of the in vivo effects of recombinant adiponectin. This paper was the foundation for many of the publications that followed.

JCI: In your career, what are you most proud of?

Scherer: Scientifically, I take great pride in the fact that we use a wide array of

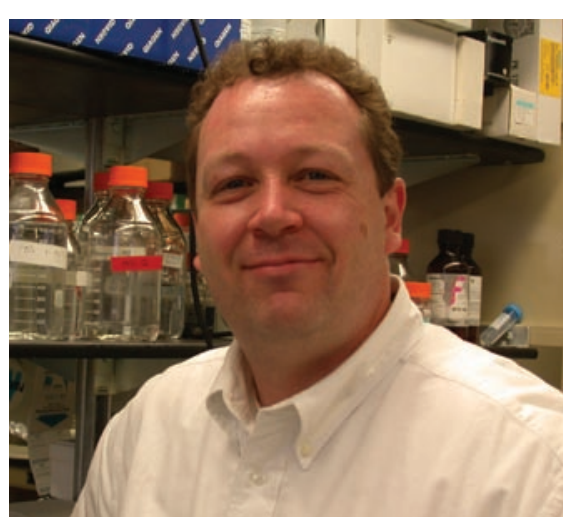

Philipp Scherer, winner of the American Diabetes Association's 2005 Outstanding Scientific Achievement Award. techniques in my laboratory to shed new light on the physiology of the adipocyte. While we use the classical molecular biology and biochemical and cell biological approaches, we are fortunate to combine this with protein structure analysis, mouse genetics, and physiology and can apply our findings directly to clinical samples. This is of course only possible through the support of a number of collaborators, who empower us to use these techniques effectively and wisely.

\section{"At the personal level, few things are more rewarding than to see a student or a postdoc grow scientifically and personally during their stay in the laboratory. I have the satisfaction that I have provided an overall environment that allowed my collaborators to achieve their full potential."}

At the personal level, few things are more rewarding than to see a student or a postdoc grow scientifically and personally during their stay in the laboratory. I have the satisfaction that I have provided an overall environment that allowed my collaborators to achieve their full potential.

JCI: What attracted you to metabolism and endocrinology?

Scherer: During my PhD, I was working on the protein translocation machinery of mitochondria. I was looking to expand my studies in a physiologically relevant system, and opted to join Harvey Lodish, who was working in the 3T3-L1 adipocyte system to study glucose transporter trafficking. I became more interested in the soluble cargo molecules of secretory vesicles in these cells. I enjoyed the fact that the proteins we were characterizing have "a life after secretion" and can have a remarkable physiological impact at the level of the whole organism.

JCI: What is your typical day like?

Scherer: No different than anybody else's day in academia. Between committee duties, teaching, and grant writing, there are often not enough hours in the day to devote just to science in the lab. I like to go home for dinner and spend time with the family, but I frequently sneak back to the lab later on to catch up on all the things that require more than a 5-minute attention span, which is the best I can achieve during regular working hours.

JCI: How do you think your work can make a difference clinically in patients with diabetes?

Scherer: I think one of the great benefits that we have in our field is the relatively short path from the bench to the bedside. Working on secreted products has the advantage that as soon as an appropriate assay is developed, clinical samples can be analyzed and the relevance of the in vitro findings can be validated. I hope that our work on adiponectin and on inflammation in adipose tissue will yield some tangible results for patients in the future.

JCI: Any parting thoughts?

Scherer: The contributions to scientific progress that we make as individuals are important. However, more than ever before, I appreciate that science is truly a group effort that requires an optimal institutional environment, the willingness of many colleagues to share thoughts and expertise, and a lot of long hours at the bench of many devoted collaborators in the lab. As such, I definitely see this prize as a reward for a group effort rather than for an individual.

\section{Stacie Bloom}

